\title{
The Strength of Relationships
}

\begin{abstract}
I endeavor to show that Descartes' attribute-mode distinction cannot be characterized in terms of the determinable-determinate relation. I identify the latter's formal and modal properties in order to determine whether the former shares them, which ultimately shows distinctness. I then indicate which property accounts for the differences. I conclude that the relation that unites modes under an attribute is weaker than that which groups determinates under some determinable, respectively, the relations of inherence and incompatibility.
\end{abstract}

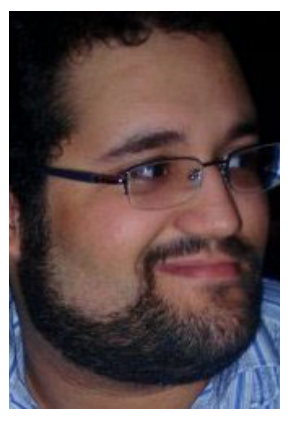

Said Saillant, a third-year student at Rutgers University (New Brunswick campus), is pursuing majors in Philosophy and Psychology and minors in Linguistics and Cognitive Science, and intends to obtain a Ph.D. in Philosophy. He is interested in the cognitive closure thesis, consciousness, the metaphysics of modality, the semantics of metaphor, theories of concepts, non-classical truth-values (e.g., gaps and gluts), the semantics of counterfactuals, and theories of conceivability. He will be presenting a paper, "The Logic of Mentalistic and Physicalistic Language," at the 2010 Pacific Division meeting of the American Philosophical Association.
$\mathrm{P}$ hilosophers have been trying to formally characterize Descartes' attribute-mode distinction for centuries. ${ }^{1}$ Throughout the years, many such proposals have been made. Somewhat recently, two characterizations have been particularly attractive, namely, the mode as trope characterization and the attribute and mode as determinable and determinate characterization. $^{2}$ The latter, more popular approach is the topic of this essay.

It is my purpose to present the determinabledeterminate relation and ascertain definitely whether it characterizes the relation between Descartes' attributes and their respective and modal properties. I then take a detour through the features of Descartes' attributemode distinction, and later contrast these with the relation between determinables and determinates in order to see whether their properties differ.

According to The Stanford Encyclopedia of Philosophy, Johnson introduces the distinction between determinables and determinates as such:

1. Edwin Curley, Descartes Against the Skeptics (Cambridge: Harvard University Press, 1978): 11; Anthony Kenny, Descartes (New York: Random House, 1968): 66; R. S. Woolhouse, Descartes, Spinoza, Leibniz: The Concept of Substance in Seventeenth Century Metaphysics (New York: Routledge, 1993):18, 26, note 3.

2. D. C. Williams, "On the Elements of Being," Review of Metaphysics 7 (1953): 186-192 ; Arthur N. Prior, "Determinables, Determinates, and Determinants (Part I)," Mind 58.229 (1949): 7, 17. 
I propose to call such terms as colour and shape determinables in relation to such terms as red and circular, which will be called determinates. ${ }^{3}$

Color is a determinable of red and circle is a determinate of shape. Immediately, a noteworthy property is apparent: the determinate contains more information than the determinable. In other words, the determinate precludes a set of possibilities greater than that precluded by the determinable.

Consequently, when predicated of some object, the determinate describes the object more precisely than its corresponding determinable because the determinate leaves fewer ways in which the object could vary. For instance, if on the one hand an object $\mathrm{O}$ is said to be colored, then all possible colors may describe $\mathrm{O}$; on the other hand, if $\mathrm{O}$ is said to be red, then only a proper subset of all possible colors may describe $\mathrm{O}$, signifying greater informational content.

This feature entails some formal properties. If $\mathrm{P}$ is a determinable of $\mathrm{Q}$, then $\mathrm{Q}$ is not a determinable of $\mathrm{P}$, for a determinable by definition leaves possibilities open that its determinates rule out. Therefore, a determinable cannot restrict the range of possibilities for one of its determinates, since the information it provides is the same regardless of whether one construes the relata as a determinable or as a determinate. To state the concept briefly, the determinable-determinate relation is asymmetric.

One other property observable at this juncture is irreflexivity. In other words, a determinable cannot be a determinable of itself and neither can a determinate be a determinate of itself. ${ }^{4}$ This is because the set of possibilities the determinate/determinable entails cannot restrict itself. The information provided would be held constant as both the determinate and the determinable; otherwise, the principle of uniform substitution would be violated and the relation would generate contradictions. ${ }^{5}$ Furthermore, it follows from the above that if $\mathrm{O}$ has a determinate $Q$, then it is necessarily the case that $\mathrm{O}$ has the determinable of $\mathrm{Q}$ because $\mathrm{Q}$ must contain the information of its determinable.

Determinates are grouped under a determinable, not because they share a certain property, but because of a "special kind of difference" that distinguishes one from another, such as the case with the grouping of red, blue, and yellow under the determinable color. ${ }^{6}$ In other words, "the determinates under a given determinable are united, not as possessors of a common character, but as a set of terms of a particular relation." 7

This particular difference is special in relation to determinates of different determinables because the determinates under one determinable cannot describe the same object at the same time. For instance, a car

3. David H. Sanford, "Determinates vs. Determinables," The Stanford Encyclopedia of Philosophy (27 October 2006) http://plato.stanford. edu/entries/determinate-determinables (20 October 2009).

4. I would like to make clear that this does not entail reflexivity (or symmetry) because Descartes is only construing thought and extension as modes insofar as a parcel of substance is capable of change, not the attribute itself, be it thought or extension, that constitutes its nature and essence.

5. Robert Goldblatt, Logics of Time and Computation (Stanford: CSLI Lecture Notes, 1992): 5.

6. Sanford.

7. Prior, 11. 
cannot be both completely red and completely blue simultaneously. Among the determinates of a determinable, there is incompatibility. Modally speaking, it is necessarily the case that two determinates under the same determinable cannot describe the same part of an object at the same time.

Moreover, being related by incompatibility is sufficient and necessary for determinates under a single determinable. ${ }^{8}$ Therefore, "for any given determinate, there is only one determinable to which it can belong." ${ }^{\prime 9}$ Given that incompatibility is necessary and sufficient to relate the determinates under a single determinable, all determinates would be grouped under a unique determinable, and as such, would preclude the possibility of those determinates belonging to any other determinable. As a consequence, it is necessarily the case that if $\mathrm{O}$ has a determinate, then $\mathrm{O}$ has the corresponding determinable. Johnson adds, "any one determinable is a literal summum genus not subsumable under any higher genus; the absolute determinate is a literal infima species under which no other determinable is subsumable." 10

One other formal property of the relation is transitivity. If $\mathrm{P}$ is a determinable of $\mathrm{Q}$ and $\mathrm{Q}$ is a determinable of $R$, then $P$ is a determinable of R. For instance, color is a determinable of blue and a determinable of navy blue, which is a determinate of blue. ${ }^{11}$ Johnson attributes another property to the relation, namely that, if a determinable $\mathrm{Q}$ is predicated of $\mathrm{O}$, then $\mathrm{O}$ must "be characterized in certain definite respect."12 In other words, if $\mathrm{O}$ is colored, then, essentially, $\mathrm{O}$ has a definite color, namely a determinate of color. The referent of $\mathrm{O}$ must be, in actuality, characterized in terms of determinates if $\mathrm{O}$ is said to have a determinable. ${ }^{13}$

Before comparing the properties ascribed to the attribute-mode relation by Descartes with those evinced by the determinable-determinate relation, I will take a tour through the relevant aspects of Descartes' philosophy. Descartes believed there were two principal properties that characterized the whole of "whatever we perceive" - thought and extension. ${ }^{14}$

Thought constitutes the nature and essence of thinking substance and extension that of corporeal substance. ${ }^{15}$ He called these primary attributes. Attributes are the properties that "always remain unchanged," such as color, shape, conation, intellection and sensation. ${ }^{16}$

For instance, some modes of extension and thought are, respectively, flavor, fragrance,

8. Ibid.

9. Sanford

10. Ibid.

11. Keep in mind that determinates do not have to be absolute and that a determinable is not a genus because it does not differentiate its members by conjoining an independent property, as happens when "man" is distinguished from other animals by conjoining animality with rationality, but by incompatibility alone.

12. Prior, 17.

13. Notice this property plus transitivity entails that a determinable $\mathrm{P}$, when predicated of $\mathrm{O}$, will, of necessity, determine its absolute determinate.

14. Cottingham, J., Stoothoff, R., \& Murdoch, D., eds. and trans. 1984. The Philosophical Writings of Descartes, vols. I-II. (Cambridge: Cambridge University Press.): 114.

15. CSM, I, 210.

16. CSM, I, 211-212. 
shape, sound and color, and imagining, doubting, understanding, affirming and perceiving..$^{17} \mathrm{~A}$ particular state of a mode can be thought to be the accidental properties of the Cartesian substances; in other words, properties it currently has but could do without. Descartes also makes clear that he believes the relation between thought and its modes and that between extension and its own are analogous. ${ }^{18}$ Consequently, whatever characterization I end up with for one would have to characterize the other.

I will select the more obscure of the two relations, namely that between thought and its modes, in an attempt to cover any possible disanalogies with the determinable-determinate relation that might not result from considering the corporeal counterpart alone. Indeed, Johnson draws many examples to illustrate his distinction from properties that Descartes would characterize as referring to extension.

An attribute is less informative when predicated of an object $\mathrm{O}$ than any of its modes would be if predicated of $\mathrm{O}$. In other words, the property of being thought entails the possibility of being any of its modes, and the property of being a particular mode $\mathrm{M}$ entails the attribute thought and the actuality of $\mathrm{M}$, thereby rendering the latter more informative. ${ }^{19}$ Therefore, the attributemode relation is asymmetric because a mode predicated of $\mathrm{O}$ eliminates at least one possibility more than an attribute would and consequently the inverse cannot hold. Moreover, irreflexivity is a property because the arguments of the relation are incompatible. An attribute is a property that precludes the possibility of change with respect to the attribute, but not with respect to its mode. ${ }^{20}$

Modes are grouped under their respective attributes because an assertion that $\mathrm{O}$ has $\mathrm{M}$ must refer to O's primary attribute in order to be intelligible. ${ }^{21}$ For instance, doubt cannot be understood independently of thought. It might seem as though we are straying from Johnson, but as Prior's property states, if a determinable $\mathrm{P}$ is predicated of $\mathrm{O}$, then $\mathrm{O}$ must have a determinate of $\mathrm{P}$, which entails that "[a subject's] being determinable in certain ways[...] is presupposed in every genuine characterization of it, an assertion that $[. .$.$] is thus determinable[...] would$ have for its predicate something which cannot really be separated from the subject in order to be predicated of it," which is precisely the property that Descartes ascribes to the attribute-mode relation. ${ }^{22}$

In Cartesian terms, if $\mathrm{O}$ has $\mathrm{M}$, then $\mathrm{O}$ has attribute A as well. This property was evidently presupposed by Descartes when he said, "whatever we find in the mind is simply one of the various modes of thinking." ${ }^{23}$ As a consequence, if $\mathrm{O}$ is a doubt, then $\mathrm{O}$ is a thought, which is the first modal property I attributed to the determinable-determinate relation. Essentially, if $\mathrm{O}$ has a determinate (a mode), then $\mathrm{O}$ has the determinable (the attribute).

The second modal property I mentioned,

17. CSM, II, 19-21.

18. Rene Descartes, "Descartes' Reply (to Arnauld's Second Letter)," in On True and False Ideas with Arnauld's New Objections to Descartes' Meditations and Descartes' Replies , ed. Elmar Kremer (Lewiston: Edwin Mellen Press, 1990): 194.

19. CSM, I, 210-211.

20. CSM, I, 211-212.

21. CSM, I, 210-211.

22. Descartes, 18 .

23. CSM, I, 210. 
however, is not so easily identified in Descartes' metaphysical motley. This property states that it is necessarily the case that two determinates under one determinable are incompatible. Descartes refers to motion and shape as modes of extension and these are clearly compatible. ${ }^{24}$ However, Descartes refers to imagination and sensory perception as modes of thought, which are incompatible. ${ }^{25}$

Perhaps incompatibility does not play a role in the attribute-mode relation; yet, in a reply to Arnauld's Second Letter of the "New Objections," Descartes asserts that "by thought I do not mean something universal which includes all modes of thinking, but rather a particular nature which receives all those modes, just as extension is a nature which receives all shapes." 26 However, in the same paragraph, Descartes explains the "just as extension . . . differs very much from the various shapes or modes of extension which it assumes, so also thought, or thinking nature, . . . is far different than this or that act of thinking [emphasis added]." 27

Descartes may be equating shape and mode, so I continue to think that incompatibility does not do much, if any, of the grunt-work for the attribute-mode relation. Moreover, Descartes says, "we are able to arrive at knowledge of one mode apart from another, and vice versa, whereas we cannot know either apart from the substance in which they both inhere." ${ }^{28}$ This is Descartes' second modal distinction. According to Descartes, this is knowledge we can only acquire via the perception of the modes themselves. ${ }^{29}$

In other words, we do not need to search for similarity or difference between modes in order to know either; we only need to know the primary attribute of the substance in which they inhere. To know or understand the attribute-mode relation, the incompatibility plays absolutely no role; whereas for the determinable-determinate relation, incompatibility is required in order to set which unique determinable determinates stand under. Two modes under one attribute may or may not describe the same part of an object at the same time.

Furthermore, in a trivial sense, supposing thought and extension were determinables, transitivity would not hold. This is because the attributes would be literal summum genus and modes absolute determinates in that they are literal infima species. In his reply to Arnauld's "New Objection," Descartes characterizes modes as the instances of thought or extension, specifically as particular acts of thinking and particular "shapes" of extension. Therefore, transitivity is not possible because there would not be a third term to which the relation may transfer. For example, if $\mathrm{A}$ is a determinable of $B$, then $B$ is a determinable of nothing else because it would be an absolute determinate. As mentioned, it would not be transitive, but only for trivial reasons.

The determinable-determinate relation differs from the attribute-mode relation in that incompatibility of modes plays no role

24. CSM, I, 214.

25.CSM, II, 54.

26. Descartes, 194.

27. Ibid.

28. CSM, I, 214.

29. CSM, II, 156. 
in determining which attribute the modes are grouped under. The relation that determines the membership of a mode to a certain attribute is that of inherence. In other words, a mode $\mathrm{M}$ is related to an attribute $\mathrm{A}$, if and only if $\mathrm{M}$ is unintelligible, without presupposing that A characterizes the substance of which $\mathrm{M}$ is a mode.

This property is evinced by the determinabledeterminate relation, formally as the transitivity relation, and modally as the property that if $\mathrm{P}$ is predicated of $\mathrm{O}$, then it is necessarily the case that $\mathrm{O}$ has the determinable of $\mathrm{P}$. Where the relations diverge is at the point Johnson introduces a stronger property which entails the relation of inherence: if $\mathrm{O}$ has a determinable $\mathrm{Q}$, then $\mathrm{O}$ necessarily has a determinate of $Q$ (the third modal property I mentioned).
As a consequence, for any determinable $\mathrm{Q}$ predicated of $\mathrm{O}$, there is only one determinate $\mathrm{P}$ that describes $\mathrm{O}$ accurately at a given timeits corresponding absolute determinate. Only one $\mathrm{P}$ can describe $\mathrm{O}$ completely at the time of predication, namely the incompatibility requirement. Therefore, the only difference is the strength of the relation that determines the determinates of a determinable versus that which determines the attribute to which a mode refers; the former is stronger than the latter. Crucially, then, the relations are differentiated in virtue of the relationship between the determinates being stronger than the relationship between modes. 